\title{
Pacific
}

Journal of

Mathematics

\section{ON ALMOST-EVERYWHERE CONVERGENCE OF INVERSE SPHERICAL TRANSFORMS}

Christopher Meaney and Elena Prestini 


\title{
ON ALMOST-EVERYWHERE CONVERGENCE OF INVERSE SPHERICAL TRANSFORMS
}

\author{
Christopher Meaney and Elena Prestini
}

\begin{abstract}
Suppose that $G / K$ is a rank one noncompact connected Riemannian symmetric space. We show that if $f$ is a bi- $K$ invariant square integrable function on $G$, then its inverse spherical transform converges almost everywhere.
\end{abstract}

\section{Introduction.}

Recall the Carleson-Hunt theorem about almost-everywhere convergence of the partial sums of the inverse Fourier transform in one dimension. If we take $1 \leq p \leq 2$ and denote by $\hat{f}$ the Fourier transform of a function $f$ in $L^{p}(\mathbb{R})$ then for each $R>0$ there is the partial sum

$$
S_{R} f(x):=\int_{-R}^{R} \hat{f}(\xi) e^{i x \xi} d \xi
$$

There is also the maximal function

$$
S^{*} f(x):=\sup _{R>0}\left|S_{R} f(x)\right|
$$

The Carleson-Hunt Theorem states that if $1<p \leq 2$ then there is a constant $c_{p}>0$ such that

$$
\left\|S^{*} f\right\|_{p} \leq c_{p}\|f\|_{p}, \quad \forall f \in L^{p}(\mathbb{R}) .
$$

When this is combined with the fact that the inverse Fourier transform converges everywhere for elements of $C_{c}^{\infty}(\mathbb{R})$, a dense subspace of $L^{p}(\mathbb{R})$, then the almost-everywhere convergence of $\left\{S_{R} f(x): R>0\right\}$ follows for all $f \in L^{p}(\mathbb{R})$. In fact, it suffices to know that there is the weak estimate on the truncated maximal operator for all $y>0$ and $f \in L^{p}(\mathbb{R})$,

$$
\left|\left\{x: \sup _{R>1}\left|S_{R} f(x)-S_{1} f(x)\right|>y\right\}\right| \leq c_{p}\|f\|_{p}^{p} / y^{p}
$$

and this follows from (3). The inequality (3) has been extended to Hankel transforms by Kanjin [4] and Prestini [6], for an appropriate interval of values for $p$. In this paper we will be concentrating on the $L^{2}$ case. 


\section{Bessel functions and Hankel transforms.}

For $\alpha>-1 / 2$ and $1 \leq p \leq 2$ consider the weighted Lebesgue space $L_{p, \alpha}(0, \infty)$ with norm

$$
\|f\|_{p, \alpha}=\left(\sigma_{\alpha} \int_{0}^{\infty}|f(x)|^{p} x^{2 \alpha+1} d x\right)^{1 / p} .
$$

Here $\sigma_{\alpha}=2 \pi^{\alpha+1} \Gamma(\alpha+1)$. Furthermore, there is the Hankel transform

$$
\tau_{\alpha} f(y)=\int_{0}^{\infty} f(x) \frac{J_{\alpha}(x y)}{(x y)^{\alpha}} x^{2 \alpha+1} d x
$$

where $J_{\alpha}$ is the usual Bessel function indexed by $\alpha$. The corresponding maximal function for the inversion of this transform is

$$
T_{\alpha}^{*} f(x)=\sup _{R>0}\left|\int_{0}^{R} \tau_{\alpha} f(y) \frac{J_{\alpha}(x y)}{(x y)^{\alpha}} y^{2 \alpha+1} d y\right| .
$$

Proposition 1 (Kanjin, Prestini). For $\alpha \geq-1 / 2$ and

$$
4(\alpha+1) /(2 \alpha+3)<p<4(\alpha+1) /(2 \alpha+1)
$$

there is a constant $c_{p, \alpha}$ such that

$$
\left\|T_{\alpha}^{*} f\right\|_{p, \alpha} \leq c_{p, \alpha}\|f\|_{p, \alpha}, \quad \forall f \in L_{p, \alpha}(0, \infty)
$$

Following the notation of $[9]$, we set

$$
\mathcal{J}_{\mu}(z):=2^{\mu-1} \Gamma\left(\mu+\frac{1}{2}\right) \Gamma\left(\frac{1}{2}\right) z^{-\mu} J_{\mu}(z) .
$$

We will make use of the following alternative formulation of the Hankel transform for $L^{2}$ spaces. Notice that $F \in L^{2}(0, \infty)$ if and only if

$$
\|F\|_{2}^{2}=\int_{0}^{\infty}\left|F(\lambda) \lambda^{-\alpha-1 / 2}\right|^{2} \lambda^{2 \alpha+1} d \lambda<\infty
$$

If $\lambda \mapsto F(\lambda) \lambda^{-\alpha-1 / 2}$ is in $L_{2, \alpha}(0, \infty)$ and $R>1$ then we can take the partial Hankel transform

(5) $\int_{1}^{R} F(\lambda) \lambda^{-\alpha-1 / 2} \frac{J_{\alpha}(\lambda t)}{(\lambda t)^{\alpha}} \lambda^{2 \alpha+1} d \lambda=t^{-\alpha-1 / 2} \int_{1}^{R} F(\lambda)(\lambda t)^{1 / 2} J_{\alpha}(\lambda t) d \lambda$. 


\section{Spherical transforms.}

3.1. Notation. First, $G$ will denote a noncompact connected semisimple Lie group. Next, we fix a maximal compact subgroup $K$ in $G$, and we assume that the rank of the symmetric space $K \backslash G$ is one. Furthermore, let $n$ be the dimension of $K \backslash G$. We assume that an Iwasawa decomposition $G=A N K$ is fixed once and for all.

Let $\mathfrak{a}$ denote the Lie algebra of $A$ inside $\mathfrak{g}$, so that $\mathfrak{a}$ is isomorphic to the real line. Following [9] we fix an element $H_{0}$ of $\mathfrak{a}$ so that $\mathfrak{a}=\mathbb{R} H_{0}$. There is the map from the real line onto $A$ defined by $a(t):=\exp \left(t H_{0}\right)$, for all real numbers $t$. Every element of $G$ can be written as $g=k_{1} a(t) k_{2}$ for some $k_{1}$ and $k_{2}$ in $K$ and $t \geq 0$. Hence, every bi- $K$-invariant function on $G$ is completely determined by its restriction to the set $\{a(t): t \geq 0\}$. There is a density $D$ on $[0, \infty)$ which corresponds to the Haar measure on $G$,

$$
\int_{G} f(x) d x=\int_{0}^{\infty} \int_{K} \int_{K} f\left(k_{1} a(t) k_{2}\right) D(t) d k_{1} d k_{2} d t
$$

for all $f \in C_{c}(G)$. Let $n$ be the dimension of the symmetric space $K \backslash G$, and let $\rho$ denote the special number described in [9].

Lemma 1. The density $D$ on $[0, \infty)$ has the properties:

$$
D(t)=O\left(t^{n-1}\right) \quad \text { as } \quad t \downarrow 0,
$$

and

$$
D(t)=O\left(e^{2 \rho t}\right) \quad \text { as } \quad t \rightarrow \infty
$$

3.2. Spherical Functions. To each complex number $\lambda$ there is associated the spherical function $\varphi_{\lambda}$, which is a smooth bi- $K$-invariant function on $G$. If $\lambda$ is real then $\varphi_{\lambda}$ is bounded and there is the spherical transform

$$
\mathfrak{F} f(\lambda):=\int_{G} f(x) \varphi_{\lambda}(x) d x
$$

for all integrable functions on $G$. If we add the hypothesis that $f$ is bi- $K$ invariant, then this reduces to a one-dimensional integral transform, namely,

$$
\mathfrak{F} f(\lambda):=\int_{0}^{\infty} f(a(t)) \varphi_{\lambda}(a(t)) D(t) d t,
$$

where $D$ is the density used in equation (1.1) of [9]. It is known that there is a density $|c(\lambda)|^{-2}$ on $[0, \infty)$ so that the spherical transform extends from being a map $\mathfrak{F}:{ }^{K} L^{1}(G)^{K} \cap L^{2}(G) \rightarrow C^{\infty}(0, \infty)$ to an isometry

$$
\mathfrak{F}:{ }^{K} L^{2}(G)^{K} \cong L^{2}\left([0, \infty),|c(\lambda)|^{-2} d \lambda\right) .
$$


This is the Plancherel theorem for bi- $K$-invariant functions [1]. It is also known that if $f \in{ }^{K} C_{c}^{\infty}(G)^{K}$ then

$$
f(x)=\lim _{R \rightarrow \infty} \int_{0}^{R} \mathfrak{F} f(\lambda) \varphi_{\lambda}(x)|c(\lambda)|^{-2} d \lambda
$$

uniformly. Let $\mathcal{S}_{R}$ denote the partial summation operator. From the results of [9] about analyticity of spherical transforms, it is clear that $\mathcal{S}_{R}$ cannot be a bounded operator from ${ }^{K} L^{p}(G)^{K}$ to ${ }^{K} L^{p}(G)^{K}$, when $p<2$. Despite this, Giulini and Mauceri have been able to treat some Riesz-Bochner means in this case, [2].

The analogue of the maximal function (2) is

$$
\mathfrak{M} f(a(t)):=\left.\sup _{R>0}\left|\int_{0}^{R} \mathfrak{F} f(\lambda) \varphi_{\lambda}(a(t))\right| c(\lambda)\right|^{-2} d \lambda\left|=\sup _{R>0}\right| \mathcal{S}_{R} f(a(t)) \mid .
$$

As we remarked above, to prove almost everywhere convergence, it is enough to consider the truncated version of this maximal function,

$$
\mathfrak{M}^{*} f(a(t)):=\left.\sup _{R>1}\left|\int_{1}^{R} \mathfrak{F} f(\lambda) \varphi_{\lambda}(a(t))\right| c(\lambda)\right|^{-2} d \lambda \mid .
$$

We wish to understand the $L^{2}$ mapping properties of $\mathfrak{M}^{*}$. This will involve estimates on $\varphi_{\lambda}(a(t))$ for all $t>0$ and large $\lambda$. These asymptotic results were found by Stanton and Tomas [9]. In [5] we use the results of Schindler[8] and direct estimates on the Dirichlet kernel to treat the case when $G=S L(2, \mathbb{R})$ and $K=S O(2)$. There we show that $\mathfrak{M}^{*}$ is bounded from ${ }^{K} L^{p}(S L(2, \mathbb{R}))^{K}$ to $L^{2}+L^{p}$, when $4 / 3<p \leq 2$.

\section{Asymptotic results.}

Theorem 2.1 of [9] gives the asymptotics of $\varphi_{\lambda}(a(t))$ for small values of $t$. In this case $\varphi_{\lambda}(a(t))$ behaves like a combination of Bessel functions.

Theorem 2. There exist $B_{0}>1$ and $B_{1}>1$ such that for all $0 \leq t \leq B_{0}$,

$$
\begin{aligned}
\varphi_{\lambda}(a(t))=c_{0}\left(\frac{t^{n-1}}{D(t)}\right)^{1 / 2} \mathcal{J}_{(n-2) / 2}(\lambda t) & \\
& +c_{0}\left(\frac{t^{n-1}}{D(t)}\right)^{1 / 2} t^{2} a_{1}(t) \mathcal{J}_{n / 2}(\lambda t)+E_{2}(\lambda, t)
\end{aligned}
$$

with $\left|a_{1}(t)\right| \leq c B_{1}^{-1}$, for all $0 \leq t \leq B_{0}$, and

$$
\left|E_{2}(\lambda, t)\right| \leq \begin{cases}c_{2} t^{4} & \text { if }|\lambda t| \leq 1 \\ c_{2} t^{4}(\lambda t)^{-((n-1) / 2+2)} & \text { if }|\lambda t|>1 .\end{cases}
$$


Similarly, they have the case for large $t$. Following Harish-Chandra [3], they write

$$
\varphi_{\lambda}(a(t))=c(\lambda) e^{(i \lambda-\rho) t} \phi_{\lambda}(t)+c(-\lambda) e^{(-i \lambda-\rho) t} \phi_{-\lambda}(t)
$$

so that

$$
\varphi_{\lambda}(a(t))=c(\lambda) e^{i \lambda t} e^{-\rho t}+c(-\lambda) e^{-i \lambda t} e^{-\rho t}+\text { error terms. }
$$

Corollary 3.9 of [9] then describes the asymptotics of the functions $\phi_{\lambda}$.

Proposition 3. For integers $M>0$ and $m \geq 0$, real numbers $t \geq B_{0}$, and real $\lambda$, there exist functions $\Lambda_{m}(\lambda, t)$ and $\mathcal{E}_{M+1}(\lambda, t)$ and a constant $A>0$ such that

$\phi_{\lambda}(t)=\Lambda_{0}(t)+\sum_{m=1}^{\infty} \Lambda_{m}(\lambda, t) e^{-2 m t}=\Lambda_{0}(t)+\sum_{m=1}^{M} \Lambda_{m}(\lambda, t) e^{-2 m t}+\mathcal{E}_{M+1}(\lambda, t)$, where $\Lambda_{0}(t) \leq A G_{0}(t)$

$$
\begin{aligned}
\left|D_{\lambda}^{\alpha} \Lambda_{m}(\lambda, t)\right| & \leq A \rho^{m} e^{2 m}|\lambda|^{-(m+\alpha)} 2^{\alpha} G_{0}(t) \\
\left|\mathcal{E}_{M+1}(\lambda, t)\right| & \leq A \rho^{M+1} e^{2(M+1)}|\lambda|^{-(M+1)} G_{0}(t) .
\end{aligned}
$$

Here $G_{0}(t)=\sum_{j=0}^{\infty} e^{2 j(1-t)}$.

The material at the top of page 260 in [9] shows that the $m=0$ term in this expansion is independent of $\lambda$ since the factors $\gamma_{0}^{k}$ used there are constant in $\lambda$. Also notice that

$$
G_{0}(t)=\sum_{j=0}^{\infty} e^{2 j(1-t)}=\frac{1}{1-e^{2-2 t}}, \quad \forall t>1 .
$$

In particular, $G_{0}$ is uniformly bounded on $\left[B_{0}, \infty\right)$.

We conclude this section by pointing out the long range behaviour of the $c$-functions, see Lemma 4.2 in [9].

Proposition 4. For real $\lambda$ and integers $\alpha \geq 0$,

$$
\left.\left|D_{\lambda}^{\alpha}\right| c(\lambda)\right|^{-2} \mid \leq c_{\alpha}(1+|\lambda|)^{n-1-\alpha} .
$$

In particular,

$$
|c(\lambda)|^{-1}=O\left(\lambda^{(n-1) / 2}\right), \quad \text { for large } \lambda .
$$

Also note that

$$
c(-\lambda)=\overline{c(\lambda)}, \quad \forall \lambda \in \mathbb{R} .
$$

This means that $c(\lambda) /|c(\lambda)|$ and $c(-\lambda) /|c(\lambda)|$ both have absolute value one. 


\section{The Main Theorem.}

Theorem 1. Suppose that $G$ is a non-compact, connected, semisimple Lie group with finite centre and real rank one, with maximal compact subgroup $K$. For every bi-K-invariant square-integrable function $f$ on $G$, the partial sums of the inverse spherical transform converge almost-everywhere on $G$.

5.1. Transplanting to one dimension. To prove this result we transplant the problem to one about Hankel and Fourier transforms. This follows an idea found in Schindler's paper [8]. If $f$ is a square-integrable bi- $K$-invariant function on $G$, set

$$
\mathfrak{R} f(t):=(D(t))^{1 / 2} f(a(t)), \quad \forall t>0 .
$$

Immediately we see that $\mathfrak{R} f \in L^{2}(0, \infty)$ and

$$
\|\Re f\|_{L^{2}(0, \infty)}=\|f\|_{L^{2}(G)}, \quad \forall f \in{ }^{K} L^{2}(G)^{K} .
$$

For real numbers $\lambda$ and $t>0$, set

$$
\psi_{\lambda}(t):=|c(\lambda)|^{-1}(D(t))^{1 / 2} \varphi_{\lambda}(a(t)),
$$

and define an integral transform on functions on $(0, \infty)$ by

$$
\mathcal{K} F(\lambda):=\int_{0}^{\infty} F(t) \psi_{\lambda}(t) d t, \quad \forall \lambda>0 .
$$

This has the properties that it is an isometry from $L^{2}(0, \infty)$ to itself and that

$$
\mathcal{K}(\Re f)(\lambda)=|c(\lambda)|^{-1} \mathfrak{F} f(\lambda), \quad \forall f \in{ }^{K} L^{2}(G)^{K} .
$$

Finally, notice that the maximal function we are interested in has the description as

$$
\mathfrak{M}^{*} f(a(t))=(D(t))^{-1 / 2} \sup _{R>1}\left|\int_{1}^{R} \mathcal{K}(\Re f)(\lambda) \psi_{\lambda}(t) d \lambda\right| .
$$

We wish to prove that if $\mathfrak{R} f \in L^{2}(0, \infty)$ then $t \mapsto(D(t))^{1 / 2} \mathfrak{M}^{*} f(a(t))$ is in $L^{2}(0, \infty)$, which is the same as asking that

$$
t \mapsto \sup _{R>1}\left|\int_{1}^{R} \mathcal{K}(\Re f)(\lambda) \psi_{\lambda}(t) d \lambda\right|
$$


be square integrable on $[0, \infty)$. We also need to estimate the norm of this in terms of the norm of $\mathfrak{R} f$.

For the moment, replace $\mathcal{K}(\Re f)$ by an arbitrary $F \in L^{2}(0, \infty)$, with the same $L^{2}$-norm. Notice that $F$ may be thought of as the restriction to $(0, \infty)$ of the Fourier transform of an element of $L^{2}(\mathbb{R})$. The results of Stanton and Tomas show that we can write $\psi_{\lambda}(t)$ in different ways, depending on the size of $t$. For $0 \leq t \leq B_{0}$ the expansion in Proposition 4 means that we have three pieces:

$$
\begin{aligned}
\psi_{\lambda}(t)=c_{0}|c(\lambda)|^{-1} t^{(n-1) / 2} \mathcal{J}_{(n-2) / 2}(\lambda t) \\
+c_{0}|c(\lambda)|^{-1} t^{2+(n-1) / 2} a_{1}(t) \mathcal{J}_{n / 2}(\lambda t) \\
+|c(\lambda)|^{-1} D(t)^{1 / 2} E_{2}(\lambda, t) .
\end{aligned}
$$

For every $B_{2}>B_{0}$ and $B_{0}<t<B_{2}$ the expansion in Proposition 4 means that we can write $\psi_{\lambda}(t)$ as

$$
\begin{aligned}
\psi_{\lambda}(t)= & \sum_{\epsilon= \pm 1}\left\{\frac{c(\epsilon \lambda)}{|c(\lambda)|} e^{\epsilon i \lambda t} e^{-\rho t} D(t)^{1 / 2} \Lambda_{0}(t)\right. \\
& +\frac{c(\epsilon \lambda)}{|c(\lambda)|} e^{\epsilon i \lambda t} e^{-\rho t-2 t} D(t)^{1 / 2} \Lambda_{1}(\epsilon \lambda, t) \\
& \left.+\frac{c(\epsilon \lambda)}{|c(\lambda)|} e^{\epsilon i \lambda t} e^{-\rho t} D(t)^{1 / 2} \mathcal{E}_{2}(\epsilon \lambda, t)\right\} .
\end{aligned}
$$

The remaining case, when $t>B_{2}>B_{0}$ is

$$
\begin{aligned}
\psi_{\lambda}(t)= & \frac{c(\lambda)}{|c(\lambda)|} e^{i \lambda t} e^{-\rho t} D(t)^{1 / 2} \Lambda_{0}(t)+\frac{c(-\lambda)}{|c(\lambda)|} e^{-i \lambda t} e^{-\rho t} D(t)^{1 / 2} \Lambda_{0}(t) \\
& +\frac{c(\lambda)}{|c(\lambda)|} e^{i \lambda t} e^{-\rho t} D(t)^{1 / 2} \sum_{m=1}^{\infty} \Lambda_{m}(\lambda, t) e^{-2 m t} \\
& +\frac{c(-\lambda)}{|c(\lambda)|} e^{-i \lambda t} e^{-\rho t} D(t)^{1 / 2} \sum_{m=1}^{\infty} \Lambda_{m}(-\lambda, t) e^{-2 m t}
\end{aligned}
$$

Later we will fix one value for $B_{2}$ depending on the values of $B_{0}, n$, and $\rho$.

- Case of small $t$, first piece. Here we must estimate

$$
T_{1}(t)=\left.\sup _{R>1}\left|\int_{1}^{R} F(\lambda)\right| c(\lambda)\right|^{-1} t^{(n-1) / 2} J_{(n-2) / 2}(\lambda t)(\lambda t)^{-(n-2) / 2} d \lambda \mid
$$

with $0 \leq t \leq B_{0}$. Notice that $|c(\lambda)|^{-1} \leq$ const. $(1+|\lambda|)^{(n-1) / 2}$ and so the function

$$
F_{1}(\lambda)=F(\lambda)|c(\lambda)|^{-1} \lambda^{-(n-1) / 2}
$$


is in $L^{2}(1, \infty)$ and $\left\|F_{1}\right\|_{2} \leq$ const. $\|F\|_{2}$. Then we must estimate

$$
\sup _{R>1}\left|\int_{1}^{R} F_{1}(\lambda)(\lambda t)^{1 / 2} J_{(n-2) / 2}(\lambda t) d \lambda\right| \text {. }
$$

See equation (5). The Kanjin-Prestini theorem implies that

$$
t \mapsto \sup _{R>1}\left|\int_{1}^{R} F_{1}(\lambda)(\lambda t)^{1 / 2} J_{(n-2) / 2}(\lambda t) d \lambda\right| \cdot t^{-(n-2) / 2-1 / 2}
$$

is in $L_{2,(n-1) / 2}(0, \infty)$ with norm less than or equal to a constant multiple of $\left\|F_{1}\right\|_{2}$, where the constant depends only on $K \backslash G$. But this means that

$$
\left(\int_{0}^{\infty}\left|T_{1}(t)\right|^{2} d t\right)^{1 / 2} \leq \text { const. }\|F\|_{2} \text {. }
$$

This completes the necessary estimate on the first part.

- Case of small $t$, second piece. Next, set $T_{2}(t)$ to be equal to

$$
\left.\sup _{R>1}\left|\int_{1}^{R} F(\lambda)\right| c(\lambda)\right|^{-1} t^{2+(n-1) / 2} a_{1}(t) J_{n / 2}(\lambda t)(\lambda t)^{-n / 2} d \lambda \mid .
$$

This can be rearranged to become

$$
\left.t a_{1}(t) \sup _{R>1}\left|\int_{1}^{R} F(\lambda) \lambda^{-1}\right| c(\lambda)\right|^{-1} \lambda^{-(n-1) / 2} J_{n / 2}(\lambda t)(\lambda t)^{1 / 2} d \lambda \mid .
$$

But $F_{2}(\lambda)=F(\lambda) \lambda^{-1}|c(\lambda)|^{-1} \lambda^{-(n-1) / 2}$ is in $L^{2}(1, \infty)$ and

$$
\left\|F_{2}\right\|_{L^{2}(1, \infty)} \leq \text { const. }\|F\|_{2} \text {. }
$$

Now apply the Kanjin-Prestini theorem to

$$
\sup _{R>1}\left|\int_{1}^{R} F_{2}(\lambda)(t \lambda)^{1 / 2} J_{n / 2}(\lambda t) d \lambda\right| .
$$

We also know that $a_{1}$ is bounded on $\left[0, B_{0}\right]$. We have proved that

$$
\left(\int_{0}^{B_{0}}\left|T_{2}(t)\right|^{2} d t\right)^{1 / 2} \leq \text { const. }\|F\|_{2} \text {. }
$$

- Case of small $t$, third piece. Set

$$
T_{3}(t)=\left.D(t)^{1 / 2} \sup _{R>1}\left|\int_{1}^{R} F(\lambda)\right| c(\lambda)\right|^{-1} E_{2}(\lambda, t) d \lambda \mid
$$


for all $0 \leq t \leq B_{0}$. From the estimates for the error term described in Proposition 4 we see that $T_{3}(t)$ is less than or equal to

$$
\begin{aligned}
& \text { const. } D(t)^{1 / 2} t^{4} \int_{1}^{1 / t}|F(\lambda) \| c(\lambda)|^{-1} d \lambda+ \\
& \text { const. } D(t)^{1 / 2} t^{2-(n-1) / 2} \int_{1 / t}^{R}|F(\lambda) \| c(\lambda)|^{-1} \lambda^{-(2+(n-1) / 2)} d \lambda .
\end{aligned}
$$

The first term is dominated by const. $D(t)^{1 / 2} t^{4}\|F\|_{2}\left(\int_{1}^{1 / t} \lambda^{n-1} d \lambda\right)^{1 / 2} \leq$ const. $D(t)^{1 / 2} t^{4}\|F\|_{2}\left(1-t^{-n}\right)^{1 / 2}$.

Recalling that $D(t)=O\left(t^{(n-1)}\right)$ as $t \rightarrow 0$, we see that this is square integrable over $\left[0, B_{0}\right]$.

For the second term, use the fact that it is dominated by

$$
\begin{aligned}
\text { const. } D(t)^{1 / 2} t^{2-(n-1) / 2} & \int_{1 / t}^{R}|F(\lambda)| \lambda^{-2} d \lambda \\
& \leq \text { const. } D(t)^{1 / 2} t^{2-(n-1) / 2}\|F\|_{2}\left(t^{3}-\frac{1}{R^{3}}\right)^{1 / 2}
\end{aligned}
$$

This shows that

$$
\left(\int_{0}^{B_{0}}\left|T_{3}(t)\right|^{2} d t\right)^{1 / 2} \leq \text { const. }\|F\|_{2}
$$

- Small $t$, summary. So far, we have shown that there is a $B_{0}>1$ and a constant $c>0$, depending on $K \backslash G$, such that for all $f$ in ${ }^{K} L^{2}(G)^{K}$,

$$
\left(\int_{0}^{B_{0}}\left|\mathfrak{M}^{*} f(a(t))\right|^{2} D(t) d t\right)^{1 / 2} \leq c\|f\|_{2} .
$$

- Case of medium size $t$ Using the results of Proposition 4 we see that if $B_{0}<t<B_{2}$, then we need to estimate terms of the form

$$
\begin{gathered}
t \mapsto \sup _{R>1}\left|\int_{1}^{R} F(\lambda) \frac{c(\lambda)}{|c(\lambda)|} e^{(i \lambda-\rho) t}(D(t))^{1 / 2} \Lambda_{0}(t) d \lambda\right| \\
t \mapsto \sup _{R>1}\left|\int_{1}^{R} F(\lambda) \frac{c(\lambda)}{|c(\lambda)|} e^{(i \lambda-\rho-2) t}(D(t))^{1 / 2} \Lambda_{1}(\lambda, t) d \lambda\right|,
\end{gathered}
$$


and

$$
t \mapsto \sup _{R>1}\left|\int_{1}^{R} F(\lambda) \frac{c(\lambda)}{|c(\lambda)|} e^{(i \lambda-\rho) t}(D(t))^{1 / 2} \mathcal{E}_{2}(\lambda, t) d \lambda\right| .
$$

We will describe the cases with $\lambda>0$, the cases where $\lambda$ is replaced by $-\lambda$ are handled in the same manner. For the term (19) note that $\lambda \mapsto c(\lambda) /|c(\lambda)|$ is a multiplier of $L^{2}$. The Carleson-Hunt theorem states that

$$
t \mapsto \sup _{R>1}\left|\int_{1}^{R} F(\lambda) \frac{c(\lambda)}{|c(\lambda)|} e^{i \lambda t} d \lambda\right|
$$

is in $L^{2}(0, \infty)$ and the norm is less than or equal to const. $\|F\|_{2}$. Recall that $\Lambda_{0}$ is bounded on $\left[B_{0}, \infty\right)$ and take into account the factor of $t \mapsto e^{-\rho t} D(t)^{1 / 2}$, which is also bounded on $\left[B_{0}, \infty\right)$.

For the term (20) we can use integration by parts, since $F$ is locally integrable. That is, write

$$
\begin{aligned}
\int_{1}^{R} F(\lambda) & \frac{c(\lambda)}{|c(\lambda)|} e^{(i \lambda-\rho-2) t} D(t)^{1 / 2} \Lambda_{1}(\lambda, t) d \lambda= \\
& D(t)^{1 / 2} e^{(-\rho-2) t} \Lambda_{1}(R, t) \int_{1}^{R} F(\lambda) \frac{c(\lambda)}{|c(\lambda)|} e^{i \lambda t} d \lambda \\
& -D(t)^{1 / 2} e^{(-\rho-2) t} \int_{1}^{R}\left(\int_{1}^{s} F(\lambda) \frac{c(\lambda)}{|c(\lambda)|} e^{i \lambda t} d \lambda\right) \frac{\partial}{\partial s} \Lambda_{1}(s, t) d s .
\end{aligned}
$$

The absolute value of these terms are less than or equal to

$$
\text { const. }(D(t))^{1 / 2} e^{(-\rho-2) t} S^{*} h(t) G_{0}(t)\left(\frac{1}{R}+\int_{1}^{R} \frac{d s}{s^{2}}\right)^{\prime}
$$

where $S^{*} h$ is the Carleson-Hunt maximal operator applied to the function $h \in L^{2}(\mathbb{R})$ with $\widehat{h}(\lambda)=F(\lambda) c(\lambda)|c(\lambda)|^{-1}$, if $\lambda \geq 1$, and zero elsewhere. We know that $\left\|S^{*} h\right\|_{2} \leq$ const. $\|F\|_{2}$. Recalling that there is a factor of $e^{-\rho t}(D(t))^{1 / 2}$ to take into account, we then see that the term (20) is in $L^{2}\left(\left[B_{0}, B_{2}\right], D(t) d t\right)$ and the norm is dominated by a constant multiple of $\|F\|_{2}$, with the constant depending on $G, B_{0}$, and $B_{2}$.

Now we concentrate on (21). The estimates in Proposition 4 show that this is dominated by

$$
\text { const. } D(t)^{1 / 2} \int_{1}^{\infty}|F(\lambda)| e^{-\rho t} G_{0}(t) \lambda^{-2} d \lambda \leq \text { const. } D(t)^{1 / 2} e^{-\rho t} G_{0}(t)\|F\|_{2} .
$$

This is clearly square integrable on intervals of the form $\left[B_{0}, B_{2}\right]$. 
- Medium $t$, summary. Now we have shown that for $B_{2}>B_{0}>1$ there is a constant $c>0$, depending on $K \backslash G$, such that for all $f$ in ${ }^{K} L^{2}(G)^{K}$,

$$
\left(\int_{B_{0}}^{B_{2}}\left|\mathfrak{M}^{*} f(a(t))\right|^{2} D(t) d t\right)^{1 / 2} \leq c\|f\|_{2} .
$$

- Case of large $t$ Here we know that

$$
\phi_{\lambda}(t)=\Lambda_{0}(t)+\sum_{m=1}^{\infty} \Lambda_{m}(\lambda, t) e^{-2 m t}
$$

with $\left|\Lambda_{m}(\lambda, t)\right| \leq A \rho^{m} e^{2 m}|\lambda|^{-m} G_{0}(t)$ and

$$
\left|\frac{\partial}{\partial \lambda} \Lambda_{m}(\lambda, t)\right| \leq A \rho^{m} e^{2 m}|\lambda|^{-1-m} 2 G_{0}(t) .
$$

If $t>B_{0}+2+\log (\rho)$, then the series above converges absolutely uniformly on intervals of the form $\left[B_{0}+2+\log (\rho)+\delta, \infty\right)$ with $\delta>0$. We have set

$$
\psi_{\lambda}(t)=\frac{c(\lambda)}{|c(\lambda)|} D(t)^{1 / 2} e^{-\rho t} e^{i \lambda t} \phi_{\lambda}(t)+\frac{c(-\lambda)}{|c(\lambda)|} D(t)^{1 / 2} e^{-\rho t} e^{-i \lambda t} \phi_{-\lambda}(t) .
$$

Take $F \in L^{2}(0, \infty)$, then to each $R>1$,

$$
\int_{1}^{R} \frac{c(\lambda)}{|c(\lambda)|} F(\lambda) D(t)^{1 / 2} e^{-\rho t} e^{i \lambda t} \phi_{\lambda}(t) d \lambda
$$

is equal to the sum

$$
\begin{aligned}
D(t)^{1 / 2} e^{-\rho t} \Lambda_{0}(t) \int_{1}^{R} \widehat{h_{1}}(\lambda) e^{i \lambda t} d \lambda & \\
& \quad+\sum_{m=1}^{\infty} D(t)^{1 / 2} e^{-2 m t-\rho t} \int_{1}^{R} \widehat{h_{1}}(\lambda) \Lambda_{m}(\lambda, t) e^{i \lambda t} d \lambda
\end{aligned}
$$

where $h_{1} \in L^{2}(\mathbb{R})$ has $\widehat{h_{1}}(\lambda)=c(\lambda)|c(\lambda)|^{-1} F(\lambda)$ for $\lambda>1$, and similarly for the $\phi_{-\lambda}$ term. The Lebesgue dominated convergence theorem justifies the interchange of integration and summation. The first part is handled directly by the Carleson-Hunt theorem. On the second part, use integration by parts on each of the summands. Since $\widehat{h_{1}}$ is locally integrable, we see that

$$
\int_{1}^{R} \widehat{h_{1}}(\lambda) \Lambda_{m}(\lambda, t) e^{i \lambda t} d \lambda
$$

is equal to

$$
-\int_{1}^{R}\left(\int_{1}^{s} \widehat{h_{1}}(\lambda) e^{i \lambda t} d \lambda\right) \frac{\partial}{\partial s} \Lambda_{m}(s, t) d s+\Lambda_{m}(R, t) \int_{1}^{R} \widehat{h_{1}}(\lambda) e^{i \lambda t} d \lambda .
$$


Taking absolute values we see that

$$
\begin{aligned}
& \left|\int_{1}^{R} \widehat{h_{1}}(\lambda) \Lambda_{m}(\lambda, t) e^{i \lambda t} d \lambda\right| \\
& \leq 2 A S^{*} h_{1}(t) G_{0}(t) \rho^{m} e^{2 m} \int_{1}^{R} s^{-1-m} d s \\
& +A S^{*} h_{1}(t) G_{0}(t) \rho^{m} e^{2 m} R^{-m}
\end{aligned}
$$

and this is less than or equal to

$$
4 A S^{*} h_{1}(t) G_{0}(t) \rho^{m} e^{2 m}
$$

for all $R>1$. From this it follows that

$$
\begin{aligned}
\mid \int_{1}^{R} \frac{c(\lambda)}{|c(\lambda)|} F(\lambda) & D(t)^{1 / 2} e^{-\rho t} e^{i \lambda t} \phi_{\lambda}(t) d \lambda \mid \\
& \leq D(t)^{1 / 2} e^{-\rho t} A G_{0}(t) S^{*} h_{1}(t) \\
& +4 A S^{*} h_{1}(t) G_{0}(t) D(t)^{1 / 2} e^{-\rho t} \sum_{m=1}^{\infty} e^{-2 m t+m \log (\rho)+2 m}
\end{aligned}
$$

We are free to take $B_{2}>B_{0}+\log (\rho)+2$ so that the sum on the right hand side is uniformly bounded for all $t>B_{2}$. The Carleson-Hunt theorem shows that

$$
\left\|S^{*} h_{1}\right\|_{2} \leq c\left\|h_{1}\right\|_{2} \leq c^{\prime}\|F\|_{2} .
$$

- Summary of the large $t$ case. Now we have shown that there exists $B_{2}>B_{0}>1$ and a constant $c>0$, depending on $K \backslash G$, such that for all $f$ in ${ }^{K} L^{2}(G)^{K}$,

$$
\left(\int_{B_{2}}^{\infty}\left|\mathfrak{M}^{*} f(a(t))\right|^{2} D(t) d t\right)^{1 / 2} \leq c\|f\|_{2}
$$

This completes the proof of the theorem. Notice that we frequently move from one $L^{2}$ function to another using the Plancherel theorem for Fourier and Hankel transforms, and we use the fact that bounded functions are multipliers of $L^{2}$. These devices are not available to us for other $L^{p}$ spaces, so that this method can only be expected to apply to the setting of $L^{2}$. 


\section{References}

[1] R. Gangolli, On the Plancherel formula and the Paley-Wiener theorem for spherical functions on semisimple Lie groups, Annals of Math., 93 (1971), 150-165.

[2] S. Giulini and G. Mauceri, Almost everywhere convergence of Riesz means on certain noncompact symmetric spaces, Ann. Mat. Pura Appl. (to appear).

[3] Harish-Chandra, Spherical functions on a semisimple Lie group I., American J. Math., 80 (1958), 241-310.

[4] Y. Kanjin, Convergence and divergence almost everywhere of spherical means for radial functions, Proc. Amer. Math. Soc., 103 (1988), 1063-1069.

[5] C. Meaney and E. Prestini, On almost everywhere convergence of the inverse spherical transform for $S L(2, \mathbb{R})$, Ark. Mat., 32 (1994), 195-211.

[6] E. Prestini, Almost everywhere convergence of the spherical partial sums for radial functions, Monatshefte Math., 105 (1988), 207-216.

[7] J. Rosenberg, A quick proof of Harish-Chandra's Plancherel theorem for spherical functions, Proc. Amer. Math. Soc., 63 (1977), 143-149.

[8] S. Schindler, Some transplantation theorems for the generalized Mehler transform and related asymptotic expansions, Trans. Amer. Math. Soc., 155 (1971), 257-291.

[9] R. J. Stanton and P. A. Tomas, Expansions for spherical functions on noncompact symmetric spaces, Acta Math., 140 (1978), 251-276.

[10] R. J. Stanton and P. A. Tomas, Pointwise inversion of the spherical transform on $L^{p}(G / K), 1 \leq p<2$, Proc Amer. Math. Soc., 73 (1979), 398-404.

Received December 1, 1992 and revised August 9, 1993. This work was partially supported by CNR and CMA.

SCHOOL OF MPCE, MACQUARIE UNIVERSITY

NSW 2109 AUSTRALIA

EMAIL ADDRESS: CHRISM@MACADAM.MPCE.MQ.EDU.AU

AND

Dipt. di Matematica, Il Universita degli studi di Roma

Via Fontanile di Carcaricola

00133 TOR VERGATA, ROMA, ITALY

EMAIL ADDRESS: PRESTINI@VAXTVM.INFN.IT 



\title{
PACIFIC JOURNAL OF MATHEMATICS
}

Founded by E. F. Beckenbach (1906-1982) and F. Wolf (1904-1989)

\section{EDITORS}

Sun-Yung Alice Chang (Managing Editor)

University of California

Los Angeles, CA 90095-1555

pacific@math.ucla.edu

F. Michael Christ

University of California

Los Angeles, CA 90095-1555

christ@math.ucla.edu

Thomas Enright

University of California

San Diego, La Jolla, CA 92093

tenright@ucsd.edu

Nicholas Ercolani

University of Arizona

Tucson, AZ 85721

ercolani@math.arizona.edu
Robert Finn

Stanford University

Stanford, CA 94305

finn@gauss.stanford.edu

Vaughan F. R. Jones

University of California

Berkeley, CA 94720

vfr@math.berkeley.edu

Steven Kerckhoff

Stanford University

Stanford, CA 94305

spk@gauss.stanford.edu
Martin Scharlemann

University of California

Santa Barbara, CA 93106

mgscharl@math.ucsb.edu

Gang Tian

Courant Institute

New York University

New York, NY 10012-1100

tiang@taotao.cims.nyu.edu

V. S. Varadarajan

University of California

Los Angeles, CA 90095-1555

vsv@math.ucla.edu

\section{SUPPORTING INSTITUTIONS}

\section{CALIFORNIA INSTITUTE OF TECHNOLOGY \\ NEW MEXICO STATE UNIVERSITY \\ OREGON STATE UNIVERSITY \\ STANFORD UNIVERSITY \\ UNIVERSITY OF ARIZONA \\ UNIVERSITY OF BRITISH COLUMBIA \\ UNIVERSITY OF CALIFORNIA \\ UNIVERSITY OF HAWAII}

\author{
UNIVERSITY OF MONTANA \\ UNIVERSITY OF NEVADA, RENO \\ UNIVERSITY OF OREGON \\ UNIVERSITY OF SOUTHERN CALIFORNIA \\ UNIVERSITY OF UTAH \\ UNIVERSITY OF WASHINGTON \\ WASHINGTON STATE UNIVERSITY
}

The supporting Institutions listed above contribute to the cost of publication of this Journal, but they are not owners or publishers and have no responsibility for its contents or policies.

Manuscripts must be prepared in accordance with the instructions provided on the inside back cover.

The Pacific Journal of Mathematics (ISSN 0030-8730) is published monthly except for July and August. Regular subscription rate: $\$ 215.00$ a year (10 issues). Special rate: $\$ 108.00$ a year to individual members of supporting institutions.

Subscriptions, orders for back issues published within the last three years, and changes of subscribers address should be sent to Pacific Journal of Mathematics, P.O. Box 4163, Berkeley, CA 94704-0163, U.S.A. Prior back issues are obtainable from Kraus Periodicals Co., Route 100, Millwood, NY 10546.

The Pacific Journal of Mathematics at the University of California, c/o Department of Mathematics, 981 Evans Hall, Berkeley, CA 94720 (ISSN 0030-8730) is published monthly except for July and August. Second-class postage paid at Berkeley, CA 94704, and additional mailing offices. POSTMASTER: send address changes to Pacific Journal of Mathematics, P.O. Box 6143, Berkeley, CA 94704-0163.

\author{
PUBLISHED BY PACIFIC JOURNAL OF MATHEMATICS at University of California, \\ Berkeley, CA 94720, A NON-PROFIT CORPORATION \\ This publication was typeset using AMS-LATEX, \\ the American Mathematical Society's TEX macro system. \\ Copyright (C) 1995 by Pacific Journal of Mathematics
}




\section{PACIFIC JOURNAL OF MATHEMATICS}

Volume $170 \quad$ No. $1 \quad$ September 1995

Generalized generalized spin models (four-weight spin models)

EIICHI BANNAI and ETSUKO BANNAI

Fine structure of the Mackey machine for actions of abelian groups with constant Mackey obstruction

SIEGFRIED ECHTERHOFF and JONATHAN ROSENBERG

The corestriction of valued division algebras over Henselian fields. I

YOON SUNG HWANG

The corestriction of valued division algebras over Henselian fields. II

YOON SUNG HWANG

The cohomology of expansive $\mathbb{Z}^{d}$-actions by automorphisms of compact, 105 abelian groups

ANATOLE KATOK and KLAUS SCHMIDT

The Anosov theorem for exponential solvmanifolds

EDWARD KEPPELMANN and CHRISTOPHER K. MCCORD

Projections of measures on nilpotent orbits and asymptotic multiplicities 161 of $K$-types in rings of regular functions. I

DONALD RAYMOND KING

On almost-everywhere convergence of inverse spherical transforms

Christopher Meaney and Elena Prestini

Characters of supercuspidal representations of $S L(n)$

Fiona AnNe Murnaghan

The cohomology of higher-dimensional shifts of finite type

KLAUS SCHMIDT

On Gorenstein surface singularities with fundamental genus $p_{f} \geqq 2$ which satisfy some minimality conditions

TADASHI TOMARU 